MEMORANDUM

RM-5109-NASA

SEPTEMBER 1966

\title{
STATISTICAL ESTIMATION PROCEDURES FOR THE "BURN-IN" PROCESS
}

Richard E. Barlow, Frank Proschan, Ernest M. Scheuer with an Appendix by Albert Madansky

GPO PRICE $\mathbf{s}$

CFSTI PRICE(S) \$

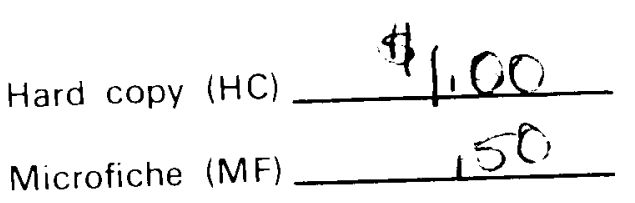

ff 653 July 65

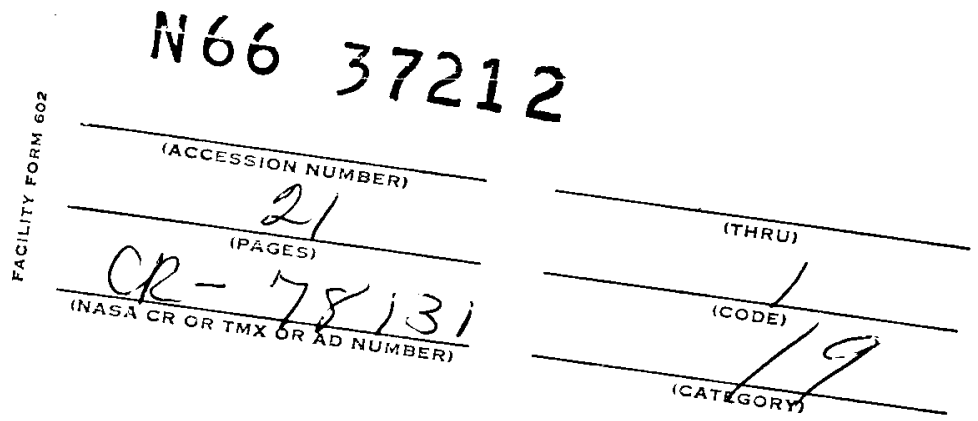

PREPARED FOR:

NATIONAL AERONAUTICS AND SPACE ADMINISTRATION 


\section{MEMORANDUM}

RM-5109-NASA

SEPTEMBER 1966

\section{STATISTICAL ESTIMATION PROCEDURES FOR THE "BURN-IN" PROCESS}

Richard E. Barlow, Frank Proschan, Ernest M. Scheuer with an Appendix by Albert Madansky

This research is sponsored by the National Aeronautics and Space Administration under Contract No. NASr-21. This report does not necessarily represent the views of the National Aeronautics and Space Administration. 
Published by The RAND Corporation 
in.

\section{RAND RESEARCH}

RM-5109-NASA, Statistical Estimation Procedures for the "Burn-in" Process, Richard E. Barlow, Frank Proschan, and Ernest M. Scheuer, with an Appendix by Albert Madansky, RAND Memorandum, August 1966, $24 \mathrm{pp}$.

PURPOSE: To consider the problem of estimating a decreasing failure-rate function from a censored or truncated sample.

APPROACH AND RESULTS: In the "burn-in" process, items whose failure rate is assumed to decrease with time are put on test (burnt-in) until a fixed amount of time has elapsed (truncated sampling) or until a fixed number of failures have occurred (censored sampling). The purpose is to identify and eliminate poorquality or defective items. There is ample evidence to show that the assumption of decreasing failure rate is valid for a variety of types of equipment.

The failure-rate function is estimated under quite general conditions. In particular, the form of the underlying life distribution need not be known. The maximum-likelihood estimate of the failure-rate function is obtained under the sole assumption that this function is decreasing. A conservative upper confidence bound on the failure rate at the end of the burn-in period is derived, assuming only that the failure rate at the time is no greater than the failure rate throughout the period of observation. The maximum-likelihood estimate and the confidence bound are obtained for both truncated and censored sampling.

BACKGROUND: This study of reliability assessment methods was done by RAND for the National Aeronautics and Space Administration's Apollo Reliability and Quality Office. It continues research reported by the authors in RM-4749-NASA, Maximum Likelihood Estimation and Conservative Confidence Interval Procedures in Reliability Growth and Debugging Problems, January 1966. 


\section{PREFACE}

\section{PRECEDING PAGE BLANK NOT FILMED.}

This Memorandum is a product of RAND's continuing interest in the estimation of reliability and related quantities. The focus here is on the "burn-in" phenomenon. The work is a continuation of research on the estimation of reliability growth and of debugging reported by the authors in RM-4749-NASA [1].

This Memorandum should be of interest to statisticians, engineers, and others concerned with various aspects of reliability.

Two of the authors, Richard E. Barlow and Frank Proschan, have been consultants to the RAND Corporation. The Appendix was written by Albert Madansky of the Market Planning Corporation, formerly a staff member of and now a consultant to The RAND Corporation. 


\section{SUMMARY}

This study deals with the "burn-in" process. In this process, items whose failure rate is assumed to decrease with time are put on test (burnt-in) until a fixed amount of time has elapsed (truncated sampling) or until a fixed number of failures have occurred (censored sampling). The purpose is to identify and eliminate poor-quality or defective items.

For both of the modes of observation described, we provide a conservative upper confidence bound for the failure rate at the time the burn-in process ends, and the maximum likelihood estimate (MLE) of the failure-rate function.

These results are valid under quite general conditions. In particular, we do not require that the form of the life distribution be known. The MLE is obtained under the sole assumption that the failure-rate function is decreasing. The confidence bound is obtained under the even weaker assumption that the failure rate at the time observation ends is no larger than the failure rate throughout the period of observation. 


\section{vii PRECEDING PAGE BLANK NOT FILMED.}

\section{CONTENTS}

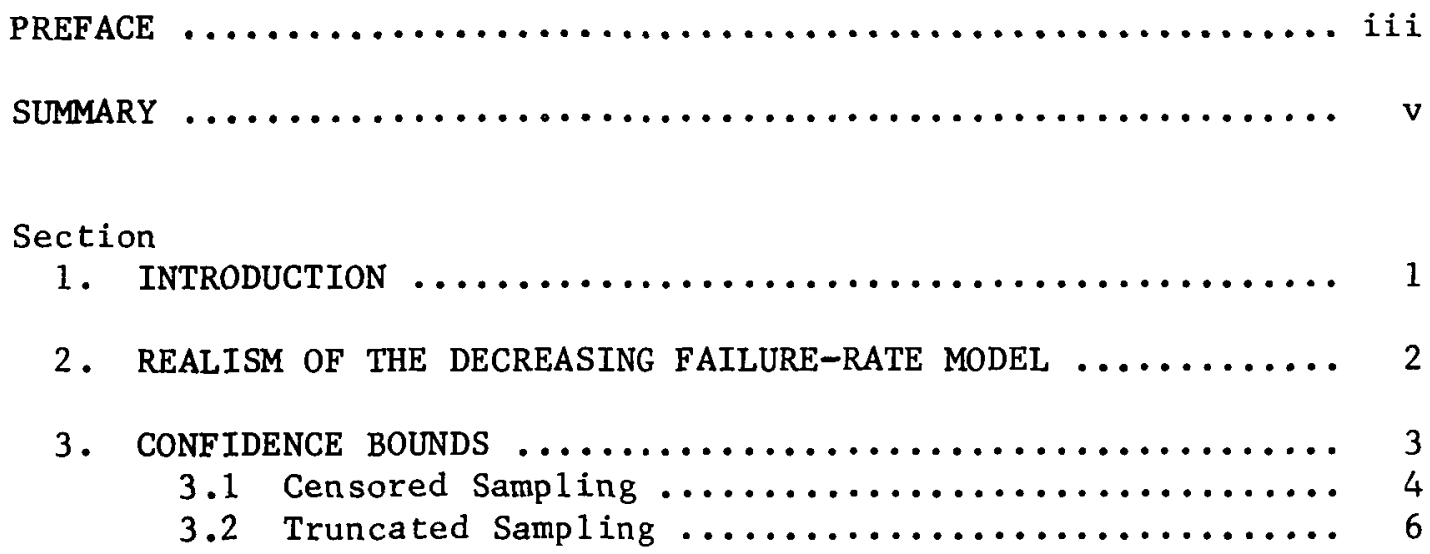

4. MLE OF A DECREASING FAILURE-RATE FUNCTION ............ 10

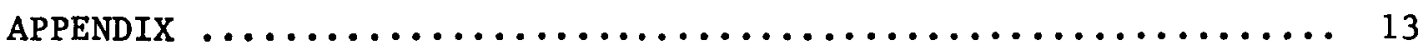

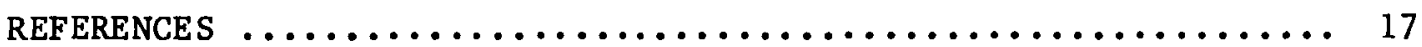




\section{INTRODUCTION}

The phenomenon of "infant mortality" has been observed in the analysis of 1 ife-test data. It arises when the failure rate is high during the early period of life and lower for a long period thereafter. The high early rate may result from contamination of the population of standard items by a small percentage of poor-quality or defective items that tend to fail soon after they are put into operation. To identify and eliminate the defective items, a practical measure often adopted is to "burn in" (i.e., put on test) all items for the period of infant mortality. From the data so obtained, it is often desired to make a confidence statement concerning the failure rate that exists after burn-in has been completed, or to obtain the maximum likelihood estimate (MLE) of the failure-rate function, or both.

This Memorandum shows how to construct such a confidence statement and the MLE based on either a truncated sample (observation ends at a fixed time, say $T$ ) or on a censored sample (observation ends after a fixed number of failures, say $r$, have occurred). Since it is a complicated matter to compute a confidence limit for the failure rate in the truncated sample case, the Appendix presents a program for machine computation developed by Albert Madansky. Examples are worked out for both censored and truncated sampling to make the results as clear as possible. To simplify the exposition, a small sample size and small (and thus possibly unrealistic) failure times were deliberately chosen for these examples. 


\section{REALISM OF THE DECREASING FAILURE-RATE MODEL}

There is ample empirical evidence to validate the model of a decreasing failure-rate function for certain classes of equipment. Mast [8] gives failure-rate curves for several types of subassemblies. His data for airline electronic and electromechanical subassemblies yield a decreasing failure rate. In a comprehensive survey, von Alven and Blakemore [11] conclude that semiconductors exhibit a decreasing failure rate. Holden [6] states that presently available data indicate that integrated circuits have a failure rate close to that of a Weibull distribution with a shape factor of 0.5 ; i.e., the failure rate for integrated circuits apparently decreases with time. 


\section{CONFIDENCE BOUNDS}

This Section derives confidence bounds on the failure rate of items surviving the burn-in period. That is, if we denote vy $\lambda$ the failure rate at the time burn-in ends (at time $T$ in the truncated sampling case, or at the time of the $r^{\text {th }}$ failure in the censored sampling case), we may make a statement of the form

$$
P[\lambda \leq U] \geq 1-\alpha
$$

The statistic $U$, a function of the observations, is called a conservative $100(1-\alpha)$ percent upper confidence bound on $\lambda$.

The following results will be needed.

THEOREM: Let (a) $\underline{Y}$ be an observation on a random variable (in general, vector-valued) having distribution function $G(y, \theta)$, with $\theta$ a one-dimensional parameter; (b) $\hat{\theta}(\underline{Y})$ be a one-dimensional statistic based on the observed vector $\underline{Y}$; (c) $\rho(\hat{\theta}(\underline{Y}))$ be a $100(1-\alpha)$ percent upper confidence bound on $\theta$, where $\dot{\rho}(u)$ is a decreasing function; (d) $\underline{X}$ be an observation on a random variable (vector-valued) having distribution function $F(\underline{x}, \theta)$; and $(e) \hat{\theta}(\underline{Y})$ be stochastically larger than $\hat{\theta}(\underline{X})$. Then

$$
P[\rho(\hat{\theta}(\underline{X})) \geq \theta \mid F(\underline{x}, \theta)] \geq 1-\alpha,
$$

that is, $p(\hat{\theta}(\underline{X}))$ is a conservative $100(1-\alpha)$ percent upper confidence bound on $\theta$, the parameter of the distribution $F$. 
Corollary: (1) If $\rho(u)$ is an increasing function and $\hat{\theta}(\underline{X})$ is stochastically larger than $\hat{\theta}(\underline{Y})$, the same result follows. (2) If $\rho(u)$ is a decreasing function, $\rho(\hat{\theta}(\underline{Y}))$ is a $100(1-\alpha)$ percent lower confidence bound on the parameter $\theta$ of $G$, and $\hat{\theta}(\underline{X})$ is stochastically larger than $\hat{\theta}(\underline{Y})$, then $\rho(\hat{\theta}(\underline{X}))$ is a conservative $100(1-\alpha)$ percent lower confidence bound on the parameter $\theta$ of F. (3) If $\rho(u)$ is an increasing function, $\rho(\hat{\theta}(Y))$ is a $100(1-\alpha)$ percent lower confidence bound on the parameter $\theta$ of $G$, and $\hat{\theta}(\underline{X})$ is stochastically smaller than $\hat{\theta}(\underline{Y})$, then $p(\hat{\theta}(\underline{X}))$ is a conservative $100(1-\alpha)$ percent lower confidence bound on the parameter $\theta$ of $\mathrm{F}$.

The theorem and its corollary are proved in Barlow, Proschan, and Scheuer [1]. These results will now be used to obtain the desired bounds. We consider both censored and truncated sampling plans.

\subsection{CENSORED SAMPLING}

Under a censored sampling plan, $n$ items are put on test (or burnt-in) and successive life-lengths $x_{1} \leq x_{2} \leq \ldots \leq x_{r}$ are observed, where $r$ is specified in advance. (If $r=n$, then the full sample has been observed.)

In the censored sampling plan for exponentially distributed lifetimes with failure rate $\lambda$, it is well known (Epstein and Sobel [3]) that an upper $100(1-\alpha)$ percent confidence limit for the failure rate, based on the censored observations $Y_{1}, Y_{2}, \ldots, Y_{r}$ is given by

$$
\frac{x_{1-\alpha}^{2}(2 r)}{2\left[\sum_{1}^{r} Y_{i}+(n-r) Y_{r}\right]}
$$

Here, as usual, $\chi_{\beta}^{2}(m)$ denotes the $100 \beta^{\text {th }}$ percentile of the chi-square distribution with $\mathrm{m}$ degrees of freedom. 
Now if $X$ is a random variable with distribution $F(\cdot)$ and failurerate function $h(\cdot)$ such that $h(t) \geq \lambda$, then $X$ is stochastically smaller than $\mathrm{Y}$, a random variable having the exponential distribution with failure rate $\lambda$. Further, the weighted sum $\left[\sum_{1}^{r} x_{i}+(n-r) x_{r}\right]$ is stochastically smaller than the corresponding weighted sum $\left[\sum_{1}^{r_{Y}} Y_{i}+(n-r) Y_{r}\right]$, so that $\hat{\theta}(X)=r /\left[\sum_{1}^{r} X_{i}+(n-r) X_{r}\right]$ is stochastically larger than $\hat{\theta}(\underline{Y})$. To use the notation of the theorem and its corollary, let

$$
\rho(\hat{\theta}(\underline{X}))=X_{1-\alpha}^{2}(2 r) \hat{\theta}(\underline{X}) / 2 r .
$$

Then by part 1 of the corollary, $p(\hat{\theta}(\underline{X}))$ is a conservative $100(1-\alpha)$ percent upper confidence bound on $\lambda$.

To sum up, if $x_{1} \leq x_{2} \leq \ldots \leq x_{r}$ are censored observations from a distribution with decreasing failure rate attaining the value $\lambda$ at time $x_{r}$, then

$$
\frac{x_{1-\alpha^{(2 r)}}^{2}}{2\left[\sum_{1}^{r} x_{i}+(n-r) x_{r}\right]}
$$

is a conservative $100(1-\alpha)$ percent upper confidence bound on $\lambda$. This result does not really require that the failure-rate function be decreasing -- only that it be bounded below by $\lambda$ over the time interval, $\left[0, x_{r}\right]$, in which observations are taken.

EXAMPLE: Consider the first 6 times to failure: $4,9,11,18$, 27,38 , assuming $\mathrm{n}=10$ items are put on test initially. A conservative $95 \%$ upper confidence bound for the failure rate at time 38 is given by 


$$
\frac{x_{.95}^{2}(12)}{2[4+9+11+18+27+38+4(38)]}=\frac{21.026}{518}=.0406
$$

a conservative $90 \%$ upper confidence bound is given by

$$
\frac{x_{.90}^{2}(12)}{518}=\frac{18.549}{518}=.0358
$$

\subsection{TRUNCATED SAMPLING}

In a truncated sampling plan, $n$ items are put on test (or burnt-in) and successive failure times $x_{1} \leq x_{2} \leq \ldots \leq x_{r}$ are observed preceding a fixed truncation (or burn-in) time $T$. The number $r$ of failures observed is random.

Bartholomew [2] has given the distribution of the MLE, $\hat{\theta}$, of the mean, $\theta$, of an exponential distribution based on a sample of $n$ truncated $^{*}$ at time $\mathrm{T}$, assuming at least one observed failure. The MLE is given by

$$
\hat{\theta}=\left[\sum_{1}^{r} x_{i}+(n-r) T\right] / r
$$

where $r$ is the number of observed failures. His result is

$$
\begin{aligned}
P_{\theta}\left[\hat{\theta} \geq \theta_{0}\right]= & \left(1-e^{-n T / \theta}\right)^{-1} \sum_{k=1}^{n}\left(\begin{array}{l}
n \\
k
\end{array}\right) \sum_{i=0}^{k}\left(\begin{array}{l}
k \\
i
\end{array}\right)(-1)^{i} \cdot \\
& \cdot \exp \left[-\frac{T}{\theta}(n-k+i)\right] \int_{X}^{\infty} p_{2 k}(u) d u,
\end{aligned}
$$

\footnotetext{
*Bartholomew uses the expression "time-censored sample" instead of "truncated sample."
} 
where $\mathrm{p}_{2 \mathrm{k}}(\cdot)$ is the chi-square density with $2 \mathrm{k}$ degrees of freedom and

$$
X=\frac{2 k}{\theta} \max \left[0, \theta_{0}-T(n-k+i) / k\right] \text {. }
$$

Albert Madansky has written a computer program to implement Bartholomew's result (see Appendix). The output of Madansky's program is a lower confidence limit for the mean 1 ife of an exponential distribution, based on a truncated sample. That is, if $\theta_{0}(\theta)$ satisfies

$$
\alpha=P_{\theta}\left[\hat{\theta} \geq \theta_{0}(\theta)\right],
$$

and if $\theta_{0}^{-1}(\cdot)$ exists and is an increasing function, then

$$
\alpha=P_{\theta}\left[\theta_{0}^{-1}(\hat{\theta}) \geq \theta\right] \text {, }
$$

or

$$
1-\alpha=P_{\theta}\left[\theta \geq \theta_{0}^{-1}(\hat{\theta})\right]
$$

Equation (9) states that $\theta_{0}^{-1}(\hat{\theta})$ is a $100(1-\alpha)$ percent lower confidence limit on $\theta$. Since, for the exponential distribution, the mean, $\theta$, and the failure rate $\lambda$, are mutually reciprocal, Eq. (9) can be rewritten to yield a $100(1-\alpha)$ percent upper confidence limit on $\lambda$ :

$$
1-\alpha=P_{\lambda}\left[\lambda \leq 1 / \theta_{0}^{-1}(\hat{\theta})\right] .
$$

Thus, Madansky's computer program provides confidence limits for the mean or, alternatively, for the failure rate of an exponential distribution, based on a truncated sample. 
To our knowledge, Madansky's program is the first to provide these 1 imits precisely. The computational difficulty of solving Eq. (9) has in the past forced dependence on approximate solutions whose accuracy is not known. The program, then, is useful for truncated sampling from exponential distributions, but our interest in it stems from its applicability to more general situations, as we will now show.

From Eq. (6) we see that $\mathrm{P}_{\theta}\left[\hat{\theta} \geq \theta_{0}\right]$ increases with $\theta$. From the definition of $\theta_{0}(\theta)(E q .(8))$ we see that for $\theta<\theta^{\prime}$,

$$
\alpha=P_{\theta}\left[\hat{\theta} \geq \theta_{0}(\theta)\right] \leq P_{\theta},\left[\hat{\theta} \geq \theta_{0}(\theta)\right] .
$$

Since $\alpha=\mathrm{P}_{\theta^{\prime}}\left[\hat{\theta} \geq \theta_{0}\left(\theta^{\prime}\right)\right]$, then $\theta_{0}(\theta) \leq \theta_{0}\left(\theta^{\prime}\right)$; that is, $\theta_{0}(\cdot)$ is an increasing function. The following diagram may clarify this argument.

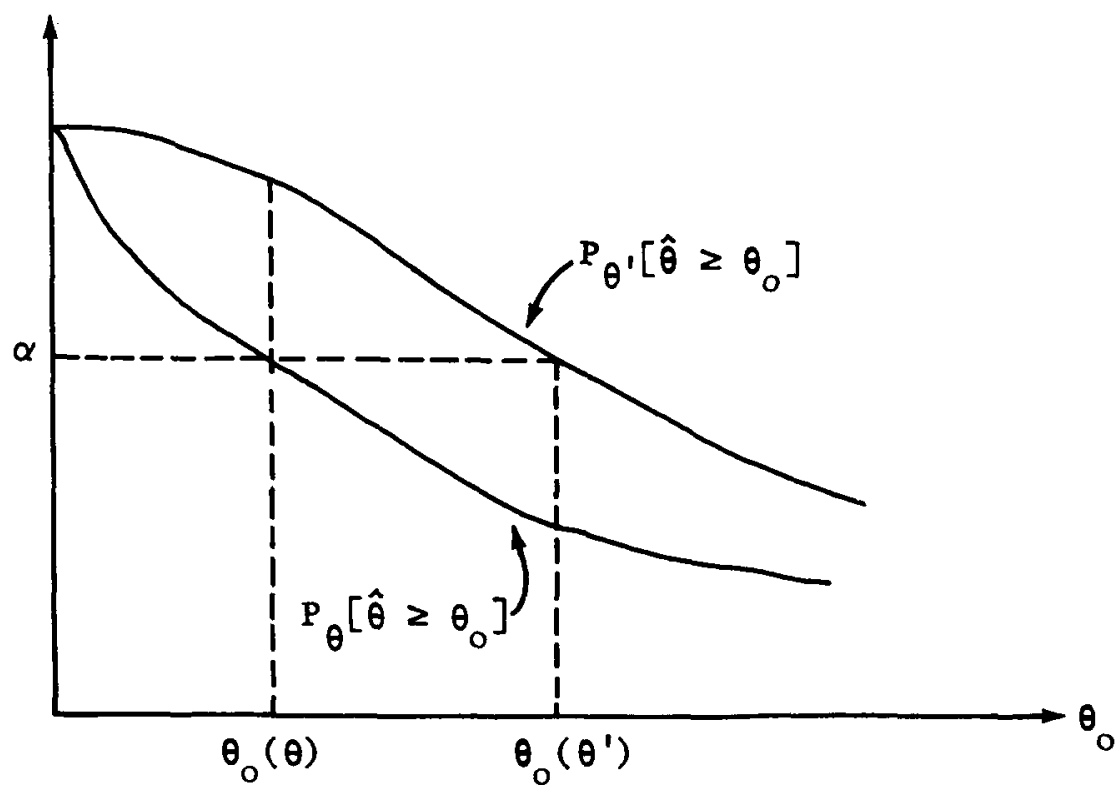

$$
\left(\theta<\theta^{\prime}\right)
$$


Thus $\theta_{0}^{-1}(\cdot)$ exists and is an increasing function, and hence $1 / \theta_{0}^{-1}(\cdot)$ exists and is a decreasing function.

Now we consider sampling from a distribution with failure-rate function $h(\cdot)$ such that for $0 \leq t \leq T, h(t) \geq h(T)=\lambda$ say. The argument of Sec. 3.1 for the censored case shows that, for each value of $r, \hat{\theta}(\underline{X})$ is stochastically smaller than $\hat{\theta}(\underline{Y})$. If we identify $p(\hat{\theta})$ with $1 / \theta_{0}^{-1}(\hat{\theta})$, we can apply our Theorem and conclude that $\rho(\hat{\theta}(\underline{X}))$ is a conservative $100(1-\alpha)$ percent upper confidence bound on $\lambda$, the failure rate at time $T$.

EXAMPLE: Suppose $\mathrm{n}=10$ items are put on test (or burnt-in) and failures are observed at times $4,9,11,18,27,38$ preceding a fixed truncation time $T=50$. We compute

$$
\hat{\theta}=\frac{1}{6}[4+9+11+18+27+38+4(50)]=51.1666 .
$$

For $\alpha=.05$ and $\alpha=.10$, respectively, Madansky's program yields .03510 and .03116 as conservative $95 \%$ and $90 \%$ upper confidence bounds on $\lambda$, the failure rate at time $T=50$. 


\section{MLE OF A DECREASING FAILURE-RATE FUNCTION}

Marshal1 and Proschan [7] have devised a procedure to obtain the MLE, $\hat{h}(\cdot)$, of a decreasing failure-rate function, $h(\cdot)$, based on a full sample. Proschan [10] has extended this procedure to more general sampling situations; this section reviews these results as they apply to censored and truncated samples.

We first describe the procedure to obtain the MLE, $\hat{h}(\cdot)$, for a censored sample. Only a slight addition is required for the case of a truncated sample, as we shall indicate.

The MLE, $\hat{h}(\cdot)$, is a step function, constant between observations, and obtained as follows. Let $z_{i}$ be the total time on test between the $(i-1)^{\text {st }}$ and the $i^{\text {th }}$ failure, $i=1, \ldots, r ; x_{1} \leq x_{2} \leq \ldots \leq x_{r}$ denote the ordered times to failure observed from the sample of size $n$; and let $x_{0}=0$. Thus, $z_{1}=n x_{1}, z_{2}=(n-1)\left(x_{2}-x_{1}\right), \ldots, z_{i}=(n-i+1)\left(x_{i}-x_{i-1}\right)$, $\ldots, z_{r}=(n-r+1)\left(x_{r}-x_{r-1}\right)$. If it happens that $z_{1}^{-1} \geq z_{2}^{-1} \geq \ldots z z_{r}^{-1}$, then

$$
\hat{h}(t)=z_{i}^{-1}, \quad x_{i-1}<t \leq x_{i}, i=1, \ldots, r
$$

If a reversal exists, i.e., for some $j, z_{j}^{-1}<z_{j+1}^{-1}$, then form the average

$$
\left[\left(z_{j}+z_{j+1}\right) / 2\right]^{-1}
$$

If

$$
z_{1}^{-1} \geq \ldots z z_{j-1}^{-1} \geq\left[\left(z_{j}+z_{j+1}\right) / 2\right]^{-1} \geq z_{j+2}^{-1} \geq \ldots z z_{r}^{-1}
$$

then 


$$
\hat{h}(t)=\left\{\begin{array}{l}
z_{i}^{-1}, x_{i-1}<t \leq x_{i}, \text { for } i=1, \ldots, j-1, j+1, \ldots, r \\
{\left[\left(z_{j}+z_{j+1}\right) / 2\right]^{-1}, x_{j}<t \leq x_{j+2} .}
\end{array}\right.
$$

If not, continue the "averaging" procedure until a decreasing sequence has been obtained. The end result will be of the form

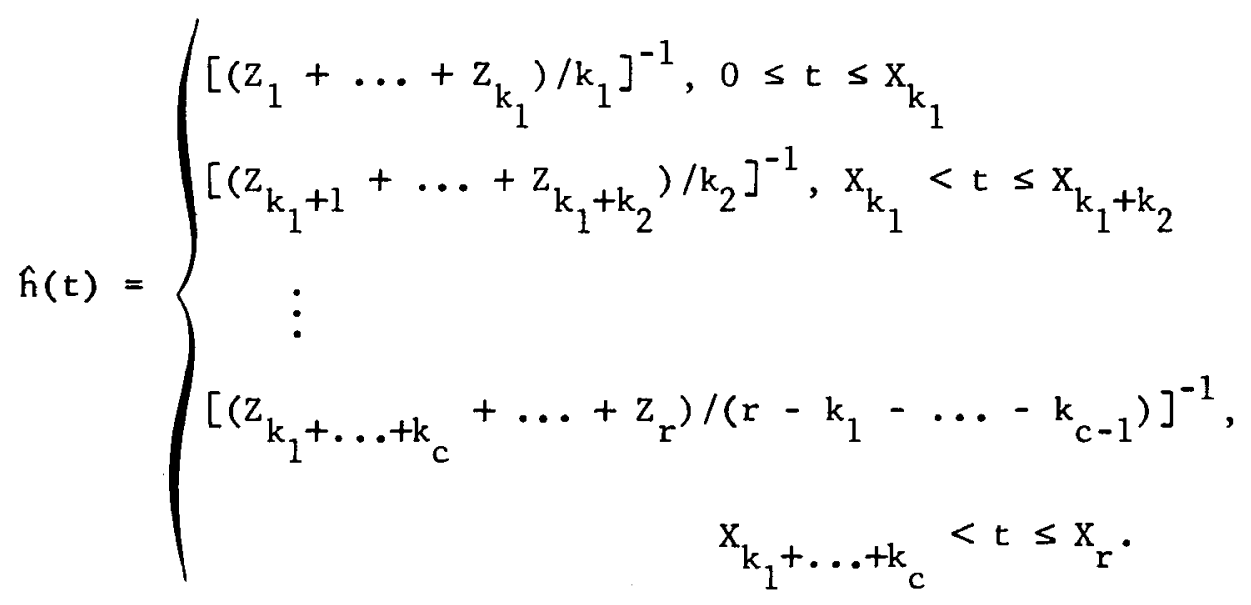

No estimate of $h(\cdot)$ is made beyond $x_{r}$ in the censored sampling case because no observations are available in that interval.

For the case of a truncated sample there is an interval $\left(\mathrm{X}_{\mathrm{r}}, \mathrm{T}\right]$ beyond the last observed failure time, $x_{r}$, in which no failure is observed. The MLE, $\hat{h}(\cdot)$, on the interval $\left[0, x_{r}\right]$ is obtained exactly as for a censored sample. For $t>x_{r}, \hat{h}(t)=0$.

EXAMPLE: Consider again the data of the examples of Sec. 3 . In a censored sample, six times to failure were observed - 4, 9, 11 , $18,27,38--$ from $n=10$ items initially put on test. We arrange the calculations for the MLE as follows. 


\begin{tabular}{l|r|c|c}
\hline$i$ & $x_{i}$ & $z_{i}=(n-i+1)\left(x_{i}-x_{i-1}\right)$ & $z_{i}^{-1}$ \\
\hline 1 & 4 & $10 \cdot 4=40$ & .0250 \\
2 & 9 & $9 \cdot 5=45$ & .0222 \\
3 & 11 & $8 \cdot 2=16$ & .0666 \\
4 & 18 & $7 \cdot 7=49$ & .0204 \\
5 & 27 & $6 \cdot 9=54$ & .0185 \\
6 & 38 & $5 \cdot 11=55$ & .0182 \\
\hline
\end{tabular}

There is a reversal between the initial estimates for the second and third intervals, so we eliminate this reversal by forming as common estimate for these intervals, $[(45+16) / 2]^{-1}=.0328$. Now there is a reversal between the estimate for the first interval and the new estimate for the second and third intervals that we just combined. We eliminate this reversal by forming, as a common estimate for these three intervals, $[(40+45+16) / 3]^{-1}=.0297$. We have now eliminated all reversals and have as the MLE of $h(\cdot)$, based solely on the assumption that $h(\cdot)$ is decreasing,

$$
\hat{\mathrm{h}}(\mathrm{t})=\left\{\begin{array}{l}
.0297, \quad 0 \leq t \leq 11 \\
.0204,11<\mathrm{t} \leq 18 \\
.0185,18<\mathrm{t} \leq 27 \\
.0182,27<\mathrm{t} \leq 38
\end{array}\right.
$$

No estimate of $h(\cdot)$ is made for $t \geq 38$, since no data are available for that interval.

In the example of Sec. 3 for a truncated sample, we supposed the same sample size and failure times as above, with continued observation, but no more failures, until the truncation time, $T=50$. The MLE in this case is given by Eq. (15) for the interval $[0,38]$, and $\hat{h}(t)=0$ for $t>38$. 
Appendix

A LOWER CONFIDENCE LIMIT FOR THE PARAMETER OF THE EXPONENTIAL DISTRIBUTION BASED ON TIME-CENSORED DATA

Albert Madansky*

In [2] Bartholomew derived the sampling distribution of $\hat{\theta}$, the maximum likelihood estimator of the mean of an exponential distribution based on time-censored data where at least one failure has been observed. In the simplest case, where $t_{1}, \ldots, t_{n}$ are independent observations on a random variable with density function

$$
f(t)=\frac{1}{\theta} e^{-t / \theta}, \quad 0 \leq t<\infty
$$

with the restriction that $t_{i}$ is observed only if $t \leq T$, the maximum likelihood estimator of $\theta$ is

$$
\hat{\theta}=\frac{1}{r} \sum_{i=1}^{n}\left\{a_{i} t_{i}+\left(1-a_{i}\right) T\right\},
$$

where

$$
a_{i}=\left\{\begin{array}{l}
1 \text { if } t_{i} \leq T, i=1, \ldots, n \\
0 \text { if } t_{i}>T, i=1, \ldots, n ;
\end{array}\right.
$$

and

$$
r=\sum_{i=1}^{n} a_{i}
$$

* The author wishes to acknowledge the stimulation of Harold Gruen, the encouragement of Ernest Scheuer, and the help of Margaret Ryan in developing this program. 
The distribution function of $\hat{\theta}$, given $r \geq 1$, is given by Eq. (6).

To obtain a lower confidence limit $\hat{\theta}_{L}$ for $\theta$ based on the distribution of $\hat{\theta}$, one must solve the equation

$$
\begin{gathered}
Y=\frac{1}{1-e^{-n T / \hat{\theta}_{L}}} \sum_{k=1}^{n}\left(\begin{array}{l}
n \\
k
\end{array}\right) e^{-(n-k)} \sum_{i=0}^{k}\left(\begin{array}{l}
k \\
i
\end{array}\right)(-1)^{i} e^{-i T / \hat{\theta}_{L}} . \\
\cdot \psi\left(2[k \hat{\theta}-(n-k+i) T] / \hat{\theta}_{L}, 2 k\right),
\end{gathered}
$$

where

$$
v(x, v)= \begin{cases}\int_{0}^{x} \frac{e^{-t} t^{v-1}}{(v-1) !} d t, & \text { if } x \geq 0 \\ 0, & \text { if } x<0\end{cases}
$$

and $Y$ is the desired confidence coefficient.

A computer program was devised to solve Eq. (18) utilizing the following features:

1) Since some iterative procedure must be utilized, the program adopts the procedure described in [9] and programmed by W. Frank in FORTRAN. In its usage at RAND the program is a subroutine called GRT, which in turn calls a subroutine AUX, which embodies the equation to be solved.

2) Our program uses as a starting value for GRT the value of $\hat{\theta}$. Perhaps better start-values, such as the lower confidence 1 imit for $\theta$ in the uncensored case, would appreciably speed up convergence, but our experience with this start-value leads us to feel that it is pragmatically adequate. 
3) To evaluate the chi-square integrals (19) in the computation of (18) we use two Hastings approximations given in [4] and [5]. Their maximum error is .0003 . These approximations are embodied in a subroutine called CHISQ.

A 1 isting of the program follows.

\$IBFTC TCFDO

C LOWFR CINFIDENCE :INIT FOR RE!IABILITY BASFD IIN

C TIME-C,ENSIIRED EXPIINENTIALLY IISTRIBUTHO DATA DIMENSIIIN X(100), C(?)

COMMON NCAP, THAT, T, GAM

READ 1,N,NCAP,T

RFAO $2,(X(1), 1=1, N)$

REAT 3,GAM

$X N=N$

$X N C, A P=N R, A P$

THAT $=(X N C A P-X N) \div 1$

$S \times I=0$.

DO $4 \mathrm{I}=1, \mathrm{~N}$

$4 S X I=S X I+X(I)$

THAT $=(S X I+$ THAT $) / X N$

$C(1)=1 . /$ THAT

CALL GRT $(1, C, I N, 0)$

$X L I M=1 . / C(1)$

PRINT 6, NCAP, N, T, GAM, THAT, XLIM

1 FORMAT (2I5, F10.5)

2 FORMAT (8F10.5)

3 FORMAT $(+10.2)$

6 FORMAT(IHO2I5,F10.5,F10.2, 2E20.8)

END

\$IRFTC AUX

SURROUTINE AUXIRT,FRT)

COMMON NCAP, THAT, T, GAM

IF(RT.GT.O.) GO TO 10

$F R T=10 . * 20$

RETUIRN

$10 \mathrm{~S}=0$.

$X N=N C A P$

$C 1=1$.

$C 2=1$.

DO $11=1$, NCAP

II $=I+1$

$X I=I$

$\mathrm{Cl}=\mathrm{Cl} *(X \mathrm{X}-\mathrm{XI}+\mathrm{L} \cdot) / \mathrm{XI}$

DO $1 K=1,11$

$X K=K-1$

IF $(K . F Q .1)$ GI) TO 6

$C ?=C ? *(\times I-X K+1=1) \times K$ 
$6 U=(X I * T H \Delta T-(X N-X I+X K) * T) * R T * 2$.

$N F=2 * I$

IF (U.LT.0.) GII TO 7

CALL CHISO (U,NF,PR)

(G) TO 8

$7 \mathrm{PR}=0$.

8 IFIAMOD $(X K, 2,1) 3,2,3$

$3 \mathrm{CN}=-1$.

(Bi) TO 9

2 $\mathrm{CN}=1$.

$9 E=E X P(-(X K+X N-X I) \neq T * R T)$

$S=S+C l * C 2 * C N N E * P K$

1 CONTINUF

$E 1=F X P(-X N * T * R T)$

$F R T=S /(1,-F 1)-G A M$

RFTURN

ENก

\$IBHTC CHISO

SIIBRIUITINE CHISQ $(11, N F, P R)$

$Y N F=N F$

$W 2=2 . / Y N F$

$W l=S O R T(W 2)$

$W 3=W 1 * W 2$

IF (U.LT.YNF)GO TO L

$T=S Q R T(11)-S D R T(Y N F)$

$A=.5-.1323 * W 1-.0036 * W 2+.0038 * 13$

$A l=.2784+.0783 * W 1-.0051 * W$ ?

$\Delta 2=.2304-.0247 * W 1-.0018 * W$ ?

$A 3=.001(1+.0592 * W 1-.0852 * W 2+.0398 * W 3$

$\Delta 4=.0781-.0906 * W L+.0923 * W 2-.0366 * W 3$

$C R=A /(1+A 1 * T+A 2 * T * 2 * A 3 * T * 3+A 4 * T * 4) * 4 * 4$

$P R=1 \cdot-C R$

RFTURN

$1 T=S D R T(Y N F / 2). * A ! O G(Y V=/ 11)$

$A=.5+.1323 * W 1+.0036 * W 2-.0038 * W 3$

$A l=.1968-.0452 * W 1-.0128 * W 2+.0067 * W 3$

$\Delta 2=.1152-.0990 * W 1+.0539 * W 2-.0168 * W 3$

$A 3=.0004+.0447 * W 1-.0866 * W 2+.0398 * W 3$

$A 4=.0195-.0629 * W 1+.0708 * W 2-.0269 * W 3$

$P K=A /(1,+A 1 * T+A 2 * T * T+A 3 * T * 3+A 4 * T * 4 * 4) * 4$

RETURN

END 


\section{REFERENCES}

1. Barlow, Richard E., Frank Proschan, and Ernest M. Scheuer, Maximum Likelihood Estimation and Conservative Confidence Interval Procedures in Reliability Growth and Debugging Problems, The RAND Corporation, RM-4749-NASA, January 1966.

2. Bartholomew, D. J., "The Sampling Distribution of an Estimate Arising in Life Testing," Technometrics, Vo1. 5, 1963, pp. 361-374.

3. Epstein, Benjamin, and Milton Sobe1, 'Life Testing," J. Am. Stat. Assoc., Vol. 48, 1953, pp. 486-502.

4. Hastings, Cecil, Jr., and Elaine Hastings, Analytical Approximations, Volume XXIV, The RAND Corporation, P-1184, September 1957.

5. ----, Analytical Approximations, Volume XXV, The RAND Corporation, P-1208, October 1957 .

6. Holden, Phil, "Proving Integrated Circuits Reliability," Proceedings of the 1966 Symposium on Reliability, San Francisco, January 25, 26, 27, 1966, pp. 464-467.

7. Marsha11, A. W., and Frank Proschan, 'Maximum Likelihood Estimation for Distributions with Monotone Failure Rate," Ann. Math. Stat., Vo1. 36, 1965, pp. 69-77.

8. Mast, L. T., Impact of Equipment Life Characteristics on Missile Test Planning, The RAND Corporation, RM-4102-PR (DDC No. AD 602646), May 1964.

9. Muller, David E., "A Method for Solving Algebraic Equations Using an Automatic Computer," Mathematical Tables and Other Aids to Computation, Vol. 10, 1956, PP. 208-215.

10. Proschan, Frank, Maximum Likelihood Estimation of a Failure Rate Initially Decreasing and then Increasing, Boeing Scientific Research Laboratories Report (in preparation).

11. Von Alven, W. H., and G. J. Blakemore, Jr., Reliability of Semiconductor Devices, Final Report, ARINC Research Corporation, $1700 \mathrm{~K} \mathrm{St.,} \mathrm{N.W.,} \mathrm{Washington,} \mathrm{D.C.,} \mathrm{Publication} \mathrm{144-6-270,}$ December 22, 1961 . 